V.E. Lashkaryov Institute of Semiconductor Physics, Nat. Acad. of Sci. of Ukraine

(41, Prosp. Nauky, Kyiv 03028, Ukraine; e-mail: koroteev@ukr.net)

PACS 85.60.-q, 07.57-c, 42.25.Bs, 42.79. $\mathrm{Pw}$

\section{THEORY OF DETECTION OF TERAHERTZ RADIATION IN HYBRID PLASMONIC STRUCTURES WITH DRIFTING ELECTRON GAS}

\begin{abstract}
The theory of non-linear interaction of electromagnetic radiation with hybrid plasmonic structure, which consists of the two-dimensional quantum heterostructure integrated with a plasmonic element in the form of a metal grating, is developed. In particular, the non-linear effect of a detection of high-frequency radiation by a drifting two-dimensional electron gas is examined. Based on the self-consistent solutions of the Maxwell and non-linear hydrodynamic equations in the frames of consistent perturbation theory of the second order, the expression of a photoresponse in the THz region is found. It is shown that the obtained expression contains an additional factor corresponding to the radiative decay rate. The latter was omitted in the previous theories. The presented theory is applied to the analysis of high-frequency properties of hybrid plasmonic structures on the basis of AlGaAs/GaAs quantum heterostructure. The influences of an optically thick substrate and the effect of the electron heating under high electron drifts on the spectral characteristics of the transmission/absorption coefficients and on the photoresponse spectra are analyzed. Some recommendations as for the design of efficient terahertz radiation detectors with the use of the hybrid plasmonic structures as a core element are given.
\end{abstract}

Ke ywords: THz plasmonics, THz detection, hybrid plasmonic structures.

\section{Introduction}

The paper is devoted to the theoretical investigation of non-linear effects arising at the interaction of terahertz $(\mathrm{THz})$ electromagnetic $(\mathrm{em})$ radiation with a strongly nonequilibrium electron gas under the excitation of plasmon oscillations. This research is closely connected to applied aspects of the further development of THz micro- and nanoelectronics.

Indeed, in spite of great successes in this field, the problem of the development of compact, highly efficient devices with electrical pumping and electrical control of working frequencies remains actual [13]. The usage of the hybrid plasmonic structures, as the core elements for such devices, is perspective and has a great potential. Their working principles are based on the resonance excitation of plasmon oscillations of a two-dimensional electron gas (2DEG) in the conductive channel of a quantum heterostructure in the presence of a plasmonic element, for instance, subwavelength metallic gratings. In this case, due to the

(C) YU.M. LYASCHUK, V.V. KOROTYEYEV, 2017

ISSN 2071-0194. Ukr. J. Phys. 2017. Vol. 62, No. 10 relatively low electron concentrations, the characteristic frequencies of plasmon oscillations lie in the $\mathrm{THz}$ range, have essential wavevector dispersion [4], and can be controlled by external applied fields. The utilization of hybrid plasmonic structures for the emission and the detection of $\mathrm{THz}$ radiation is widely discussed in the literature.

Recently, an electrically driven thermal $\mathrm{THz}$ radiation source based on $\mathrm{AlGaN} / \mathrm{GaN}$ heterostructure with micrometer scaled grating was demonstrated [5]. It has a specific form of the emission spectrum associated with a radiative decay of $2 \mathrm{D}$ plasmons [6]. In addition, the possibilities of the development of coherent $\mathrm{THz}$ sources, by using the effects of Dyakonov-Shur [7-9] and Cherenkov's [10-12] plasma instabilities induced by strong electric fields, were discussed. However, these ideas have not been verified reliably in experiments, for the present.

At the same time, the greater progress is achieved in the problem of $\mathrm{THz}$-wave detection. The modern semiconductor receivers of $\mathrm{THz}$ radiation are the square-law detectors, which transform the high- 
frequency signal to a steady-state $(d c)$ signal (photoresponse) proportional to the intensity of radiation. The physical asymmetry and/or strong nonlinearity of electrical characteristics of such devices are required for the detection of the external radiation. The asymmetry can be structural and builtin on the device fabrication stage (asymmetry of the contact system, modulation of the electron density $[13,14])$ or induced by an external electric field [15]. For example, the electrical asymmetry of contacts in the nanoscale Field-Effect Transistors (FET) $[13,16,17]$ or the size-effect of carrier depletion in self-switching diodes (SSD) $[18,19]$ is used for a detection of $\mathrm{THz}$ signals.

In the field of $\mathrm{THz}$ detectors, the great attention is paid to silicon metal-oxide-semiconductor-FETs (MOSFETs) structures. From the point of view of applications, the silicon MOSFETs are the most important ones due to the well-developed fabrication and integration in electrical circuits. It should be noted that the mechanism of detection of high-frequency radiation in such structures has a non-resonant character and is not directly associated with the excitation of plasmon modes. The mechanism of nonresonant detection is realized, when the carrier relaxation times are much shorter than the time periods of oscillations of a plasmon and an external signal. The typically non-resonant detection is described by the broadband spectral sensitivity, and it was intensively studied for different silicon transistor structures [2023]. Particularly, for the silicon MOSFET structure, the values of detected drain-source photovoltage of order of $\mathrm{mV}[21,22]$ in sub- $\mathrm{THz}$ frequency range were reported. In this case, the values of photoresponse of several tens of $\mathrm{V} / \mathrm{W}$ with a noise equivalent power of $10^{-10} \mathrm{~W} / \mathrm{Hz}^{1 / 2}[24]$ were obtained. Nowdays, the arrays of silicon detectors supplied with special antenna exhibit the superior sensitivity up to several $\mathrm{kV} / \mathrm{W}[25,26]$ and are used as a core element for THzimaging setups.

However, the high electron mobility transistor (HEMT) structures with the possibility of the plasmon mechanism of resonant detection remain still actual due to the enhanced functional abilities. Particularly, the electrical control of the spectral sensitivity of a detector can be used to obtain a colored imaging from a single array of detectors. The first experimental evidences of the plasmon mechanism of resonant detection were demonstrated for
GaAs HEMT [27]. The device detected the signal with a frequency of $2.5 \mathrm{THz}$, which was much higher than the transistor cutoff frequency. This fact was a result of the resonant excitation of plasmon modes in the channel of a transistor. Later, the mechanism of resonant detection of $\mathrm{THz}$ radiation was identified for various HEMTs based on InGaAs/InP [28], InGaAs/InAlAs [29] and InGaAs/GaAs [30] heterostructures.

The nanometer size of the FET and HEMT structures is the main disadvantage that strongly restricts their application to the detection of $\mathrm{THz}$ radiation. Typically, the size of a transistor is much more smaller than the size of the cross section of a $\mathrm{THz}$ beam, i.e., only a small part of the incident radiation is transformed to the useful signal. To increase the effective coupling of the external $\mathrm{THz}$ radiation with plasmon excitations, it is necessary to match the transistor with an antenna. This circumstance sophisticates the fabrication of the arrays of $\mathrm{THz}$ detectors based on nanoscale transistors, where each transistor should be supplied by the own antenna.

This problem can be solved by the use of a hybrid plasmonic structure with subwavelength metallic grating. The metallic grating plays the role of a broadband antenna that can effectively couple incident radiation with plasmon oscillations in the conductive channel of quantum heterostructures. The advantages of such plasmonic structure are the large effective area of a detector (the novel methods of extreme ultraviolet interference lithography allow one to easily fabricate the large-area structured surface with deeply submicron resolution $[31,32]$ ) and frequency and polarization selectivities. The selectivity is stipulated by the resonance absorption of $\mathrm{THz}$ radiation due to the excitation of plasmons in a hybrid plasmonic structure with metallic grating.

For the first time, the detection of $\mathrm{THz}$ radiation with the use of a hybrid plasmonic structure was observed in Ref. [33]. The plasmonic structure was fabricated on the basis of a double quantum well (QW) AlGaAs/GaAs heterostructure and had sensitivity of order of $1 \mathrm{mV} / \mathrm{W}$ at $25 \mathrm{~K}$ [33]. In the further experiments with the use of single QW heterostructures with micron grating periods, the values of photoresponse up to one hundred of $\mathrm{mV} / \mathrm{W}[35,36]$ were reached.

The modern theories of detection of $\mathrm{THz}$ radiation in hybrid plasmonic structures with 2DEG consider

ISSN 2071-0194. Ukr. J. Phys. 2017. Vol. 62, No. 10 
two main mechanisms: appearance of the photoresponse induced by the electron drift of 2DEG under the applied $d c$ voltage [15] and the emergence of a photoresponse due to the spatial modulation of electron concentrations in structures with the asymmetric grating-gates $[14,37,38]$. These theories are based on the perturbative solutions of the nonlinear system of equations that includes the hydrodynamic and Maxwell's equations. Their solution allowed one to obtain the expression for a photoresponse. However, from the mathematical point of view, the theories have an essential shortcoming. In the frames of the proposed approach, the hydrodynamic equations were considered in the second order of perturbation theory, but the solutions of Maxwell's equations were obtained in a linear approximation. This led to the violation of the complete self-consistency of those theories.

The aim of this paper is the development of a consistent nonlinear theory of the detection of a highfrequency radiation in hybrid plasmonic structures with the drifting 2DEG. The theory will include a consideration of the multilayer geometry of a plasmonic structure with regard for the effects of electron heating under the action of strong enough applied $d c$ electric fields.

The paper is organized as follows. Section 2 together with the Appendices includes the model, mathematical formulation of the perturbation theory of a detection, and the consistent procedure of obtaining the basic expression of the photoresponse. The developed theory is applied to the analysis of the spectral dependences of the photoresponse of a plasmonic structure based on AlGaAs/GaAs quantum heterostructure. The main results and their analysis are presented in Section 3. Particularly, the behavior of the transmission/absorption spectra and their correlation with photoresponse spectra in dependence on the geometric parameters of a plasmonic structure and the magnitude of the applied $d c$ electric field are studied. The conclusions are summarized in Section 4.

\section{Theory of Detection}

\subsection{Model and basic equations}

The model of hybrid plasmonic structure is shown in Fig. 1 and consists of the quantum heterostructure with 2DEG growing on the dielectric substrate. The metallic grating with period $a$ is deposited on the

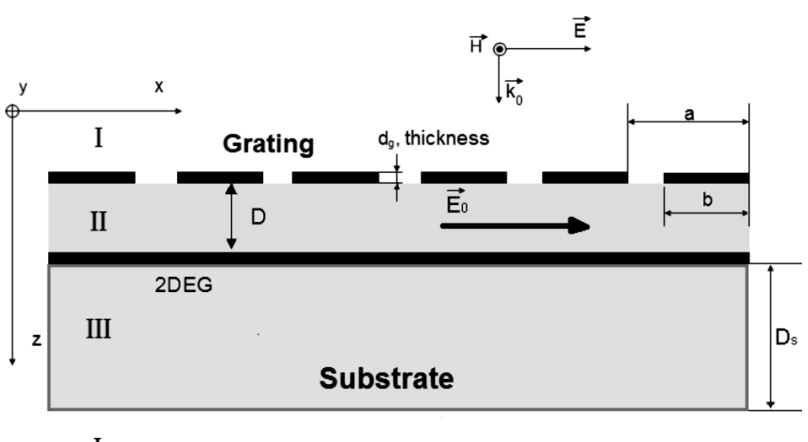

I

Fig. 1. Sketch of the geometry of the hybrid plasmonic structure based on a quantum heterostructure with $2 \mathrm{DEG}$. The $D$ and $D_{s}$ are the thicknesses of the barrier layer and substrates, respectively. Three vectors $\mathbf{k}_{0}, \mathbf{E}$, and $\mathbf{H}$ denote the orientations of the wavevector and vectors of electric and magnetic components of the incident $\mathrm{em}$ wave, respectively. The vector $\mathbf{E}_{0}$ shows the directions of $d c$ applied electric field

top surface of the barrier layer. The grating is formed by parallel strips of a metal with thickness $d_{g}$ and width $b$. We consider a deeply subwavelength grating, $a \ll \lambda_{0}$, with $\lambda_{0}$ denoting the wavelength of the incident $e m$ wave. We assume the case of the normal incidence of a plane monochromatic $(\mathbf{E}(t) \sim \exp (-i \omega t))$ em wave with its polarization oriented perpendicularly to the grating strips. In such geometry, the electric field of the $\mathrm{em}$ wave induces instantaneous dipoles, which form a spatially inhomogeneous field, in the grating strips. This field can efficiently excite the plasmon oscillations in 2DEG. To ensure the strong coupling between the external wave and plasmons in 2DEG, the thickness of the barrier layer, $D$, should be essentially less than $a$.

The coordinate system of the problem is the following: the axis $O X$ is directed along the grating axis (perpendicularly to the grating strips), and the axis $O Z$ is directed along the wavevector of the incident wave. It is assumed that, along the $O Y$ axis, the structure is uniform and infinitely long. In this case, the electric field components of the $\mathrm{em}$ wave depend on two coordinates $x$ i $z$, i.e., the electrodynamics of the problem is effectively two-dimensional. In the farfield zone of the structure $(z \gg a)$, the electric field has only one component, $E_{x}(z)$. In the near-field zone $(z \sim a)$, due to the interaction of the incident wave with the grating, the field acquires a complicated vector geometry, which is described by two components, $E_{x}(x, z)$ and $E_{z}(x, z)$ [39]. 
For the analysis of the interaction of an external electromagnetic field with the plasmonic structure, we use Maxwell's equations written in the form of the single wave equation:

$\operatorname{rot} \operatorname{rot} \mathbf{E}(\mathbf{r}, t)+\frac{\epsilon(\mathbf{r})}{c^{2}} \frac{\partial^{2} \mathbf{E}(\mathbf{r}, t)}{\partial t^{2}}=-\frac{4 \pi}{c^{2}} \frac{\partial \mathbf{j}(\mathbf{r}, t)}{\partial t}$,

where $c$ is the light velocity in vacuum, $\epsilon(\mathbf{r})$ is the local dielectric permittivity of the medium, and $\mathbf{j}(\mathbf{r}, t)$ is the high-frequency $(a c)$ conduction current density, which includes the current density in the grating, $\mathbf{j}^{G}(\mathbf{r}, t)$, and in the layer of $2 \mathrm{DEG}, \mathbf{j}^{2 \mathrm{D}}(\mathbf{r}, t)$. For the assumed geometry of the plasmonic structure, $\epsilon(\mathbf{r})$ is the function only of the $z$-coordinate,

$\epsilon(z)= \begin{cases}\epsilon_{I}, & z<0 \\ \epsilon_{I I}, & 0<z<D \\ \epsilon_{I I I}, & D<z<\mathfrak{D}, \\ \epsilon_{I}, & z>\mathfrak{D},\end{cases}$

where $\mathfrak{D}=D+D_{s}$ is the distance between the grating and the bottom interface of the substrate. Let us assume that the thicknesses of the grating strips and the region of $2 \mathrm{DEG}$ are much less than the corresponding skin layer for the electromagnetic waves with $\mathrm{THz}$ frequencies. Under this assumption, the current density will have only the nonzero $x$-component that can be written as follows:

$j_{x}(\mathbf{r}, t)=j^{G}(x, t) \delta(z)+j^{2 \mathrm{D}}(x, t) \delta(z-D)$,

where $\delta$ denotes the Dirac delta-function. To find the current in the grating, we use Ohm's law in the local approximation,

$j^{G}(x, t)=\sigma^{G}(x) E_{x}(x, 0, t)$,

where the functional dependence of the $2 \mathrm{D}$ grating conductivity $\sigma^{G}(x)$ is determined by the spatial grating profile. We assume that the conductivity of metal strips has no frequency dispersion (it is realized for the majority of highly conductive metals in the $\mathrm{THz}$ frequency range [40]). The frequency dispersion of the conductivity of noble metals can be essential in the visible range of electromagnetic waves. In this case, the conductivity becomes a complex-valued function that can modify the electrodynamic properties of a metallic grating. In particular, it can give rise to the strong absorption of electromagnetic waves and the emergence of the plasmon resonance in a grating $[41,42]$.
The determination of the current $j^{2 \mathrm{D}}(x, t)$ requires the specification of the electron transport model in 2DEG. Here, we apply the hydrodynamic model (applicability of the hydrodynamic approach is discussed in Ref. [7]) which includes: the Euler equation,

$$
\begin{aligned}
& \frac{\partial v(x, t)}{\partial t}+v(x, t) \frac{\partial v(x, t)}{\partial x}= \\
& =-\frac{e}{m^{*}} E_{x}(x, D, t)-\gamma v(x, t),
\end{aligned}
$$

the continuity equation,

$\frac{\partial n(x, t)}{\partial t}-\frac{1}{e} \frac{\partial j^{2 \mathrm{D}}(x, t)}{\partial x}=0$

and the current density in the local approximation:

$j^{2 \mathrm{D}}(x, t)=-e n(x, t) v(x, t)$,

where $e$ is the elementary charge, $m^{*}$ is the electron effective mass (the parabolic dispersion law is assumed for electrons), $n(x, t)$ and $v(x, t)$ are the electron local concentration and the average velocity, respectively. The quantity $E_{x}(x, D, t)$ is the electric field in the plane of 2DEG. The electron scattering processes are taken into account by the parameter $\gamma=1 / \tau$ that has a meaning of the momentum scattering rate.

The essentially non-linear system of equations (1)(7) will be solved in the frame of perturbation theory. For this purpose, all entering variables are expanded in series as follows:

$$
\begin{aligned}
& n(x, t)=n_{0}+n^{(1)}(x, t)+n^{(2)}(x, t), \\
& v(x, t)=v_{0}+v^{(1)}(x, t)+v^{(2)}(x, t), \\
& E_{\{x, z\}}(x, z, t)=E_{0}+E_{\{x, z\}}^{(1)}(x, z, t)+ \\
& +E_{\{x, z\}}^{(2)}(x, z, t), \\
& j_{x}(x, z, t)=j_{0}+j_{x}^{(1)}(x, z, t)+j_{x}^{(2)}(x, z, t) .
\end{aligned}
$$

The quantities $n_{0}, v_{0}, E_{0}$, and $j_{0}$ correspond to the uniform $d c$ electron concentration, drift velocity, and current density in 2DEG, respectively. The superscripts (1), (2) enumerate the terms, which are proportional to the first and second degrees of the amplitude of the external wave. We restrict ourselves by the consideration of the second-order perturbation theory, and the terms of the orders higher than 2 will be omitted. We begin the analysis of system (1)-(7) with the wave equation. The linearity of the wave equation

ISSN 2071-0194. Ukr. J. Phys. 2017. Vol. 62, No. 10 
with respect to the electric field allows us to find its solution for the different orders of a perturbation separately.

Due to the periodicity of the plasmonic structure along the $O X$ axis, we can search for the solution of Eq. (1) in the form of the Fourier expansion in the coordinate $x$. Moreover, the non-linear terms in the hydrodynamic equations can give rise to the non-linear effect such as the emergence of multiple harmonics in the frequency spectra of the $a c$ fields/currents. Thus, the electric field components of the electromagnetic wave and current densities can be expressed in the following form:

$$
\begin{aligned}
& {\left[E_{\{x, z\}}^{(s)}(\mathbf{r}, t), j_{x}^{(s)}(\mathbf{r}, t)\right]=} \\
& =\sum_{m, p=-\infty}^{+\infty}\left[E_{\{x, z\}, m, p}^{(s)}(z), j_{x, m, p}^{(s)}(z)\right] \exp \left(i\left[q_{m} x-\omega_{p} t\right]\right),
\end{aligned}
$$

where $q_{m}=2 \pi m / a, \omega_{p}=\omega p, \omega$ is the frequency of the incident signal, and superscript $s=(1,2)$ is the order of perturbation. With the use of expansion (9), the wave equation (1) can be transformed to a system of differential equations with respect to the coordinate $z$ (in the each medium) for the each $m, p$ Fourier harmonic of the $x$-component of the electric field:

$$
\begin{aligned}
& \frac{\partial^{2} E_{x, m, p}^{(s)}}{\partial z^{2}}-k_{r, m, p}^{2} E_{x, m, p}^{(s)}=\frac{4 \pi i k_{r, m, p}^{2}}{\epsilon_{r} \omega_{p}}\left[j_{m, p}^{G(s)} \delta(z)+\right. \\
& \left.+j_{m, p}^{2 \mathrm{D}(\mathrm{s})} \delta(z-D)\right]
\end{aligned}
$$

where subscript $r=I, I I, I I I$ enumerates the three media: vacuum, the barrier layer, and a substrate (see Fig. 1). The characteristic wavenumber

$k_{r, m, p}= \begin{cases}\sqrt{q_{m}^{2}-\epsilon_{r}\left(\frac{\omega_{p}}{c}\right)^{2}}, & q_{m}>\sqrt{\epsilon_{r}} \omega_{p} / c, \\ -i \sqrt{\epsilon_{r}\left(\frac{\omega_{p}}{c}\right)^{2}-q_{m}^{2}}, & q_{m}<\sqrt{\epsilon_{r}} \omega_{p} / c\end{cases}$

describes the spatial localization of the $m, p$-Fourier harmonics, which compose the spatial geometry and temporal dynamics of the resultant electromagnetic fields.

Solving Eqs. (10) with the appropriate boundary conditions at the interfaces between different media, we can find the relation between the $a c$ electric field in the plane of 2DEG and the ac currents that are induced in the plasmonic structure for the different orders of a perturbation:

$$
\begin{aligned}
& E_{x, m, p}^{(s)}(D)=-\frac{4 \pi i k_{I, m, p}}{\omega_{p} \epsilon_{I}}\left(j_{m, p}^{G(s)}+\Lambda_{m, p} j_{m, p}^{2 \mathrm{D}(\mathrm{s})}\right) \frac{1}{\Delta_{m, p}}+ \\
& +2 \tilde{E}_{0} \delta_{m, 0} \delta_{p, 1} \frac{\delta_{s, 1}}{\Delta_{m, p}}
\end{aligned}
$$

where $\tilde{E}_{0}$ is the amplitude of the incident wave. The details of the procedure of solution of Eq. (10) and the explicit form of the entering functions $\Lambda_{m, p}, \Delta_{m, p}$ are given in Appendix 1. Relation (11) will be used to determine the photoresponse.

\subsection{Expression for the photoresponse}

To obtain a formula for the photoresponse, we apply the expansions of perturbation theory (8) to the hydrodynamic equations (5)-(7). Note that all quantities are referred to $z=D$. In the zero order, it is easy to obtain the relations between $d c$ characteristics, $v_{0}=-\mu_{0} E_{0}, j_{0}=\sigma_{0} E_{0}$, where $\mu_{0}=e / m^{*} \gamma$ and $\sigma_{0}=e n_{0} \mu_{0}$ are the electron mobility and conductivity of $2 \mathrm{DEG}$, respectively. In the linear approximation, system (5)-(7) takes the form

$$
\begin{aligned}
& \frac{\partial v^{(1)}}{\partial t}+v_{0} \frac{\partial v^{(1)}}{\partial x}=-\frac{e}{m^{*}} E_{x}^{(1)}-\gamma v^{(1)} \\
& \frac{\partial n^{(1)}}{\partial t}+v_{0} \frac{\partial n^{(1)}}{\partial x}+n_{0} \frac{\partial v^{(1)}}{\partial x}=0 \\
& j^{2 \mathrm{D}(1)}=-e\left(n_{0} v^{(1)}+n^{(1)} v_{0}\right) .
\end{aligned}
$$

From system (12) written in the Fourier representation

$$
\begin{aligned}
& \left(\gamma-i\left[\omega_{p}-q_{m} v_{0}\right]\right) v_{m, p}^{(1)}=-\frac{e}{m^{*}} E_{x, m, p}^{(1)}, \\
& \left(\omega_{p}-q_{m} v_{0}\right) n_{m, p}^{(1)}-q_{m} n_{0} v_{m, p}^{(1)}=0, \\
& j_{m, p}^{2 \mathrm{D}(1)}=-e\left(n_{0} v_{m, p}^{(1)}+n_{m, p}^{(1)} v_{0}\right),
\end{aligned}
$$

we can find the $a c$ conductivity of $2 \mathrm{DEG}, \sigma_{m, p}^{2 \mathrm{D}}$, which describes, in the linear approximation, the response of the drifting 2DEG on the external spatially nonuniform $a c$ signal, i.e., $j_{m, p}^{2 \mathrm{D}(1)}=\sigma_{m, p}^{2 \mathrm{D}} E_{x, m, p}^{(1)}$ with

$\sigma_{m, p}^{2 \mathrm{D}}=\sigma_{0} \frac{\omega_{p}}{\omega_{p}-q_{m} v_{0}} \frac{\gamma}{\gamma-i\left(\omega_{p}-q_{m} v_{0}\right)}$.

The asymmetry of this quantity (at non-zero $v_{0}$ ) with respect to the substitution $q_{m}->q_{-m}$ is the responsible for the specific splitting of the plasmon resonance in the transmission and absorption spectra (see 
Section 3). For the further analysis, it is convenient to express the $m, p$-Fourier harmonics of a local electron concentration and average velocity through the $m, p$-Fourier harmonics of an $a c$ field in the plane of 2DEG

$$
\begin{aligned}
& v_{m, p}^{(1)}=-\frac{e}{m^{*}} \frac{E_{x, m, p}^{(1)}}{\left(\gamma-i\left[\omega_{p}-q_{m} v_{0}\right]\right)}, \\
& n_{m, p}^{(1)}=-\frac{e n_{0}}{m^{*}} \frac{q_{m} E_{x, m, p}^{(1)}}{\left(\gamma-i\left[\omega_{p}-q_{m} v_{0}\right]\right)\left(\omega_{p}-q_{m} v_{0}\right)} .
\end{aligned}
$$

In the quadratic approximation, the hydrodynamic equations take the following form:

$$
\begin{aligned}
& \frac{\partial v^{(2)}}{\partial t}+v_{0} \frac{\partial v^{(2)}}{\partial x}+v^{(1)} \frac{\partial v^{(1)}}{\partial x}=-\frac{e}{m^{*}} E_{x}^{(2)}-\gamma v^{(2)} \\
& \frac{\partial n^{(2)}}{\partial t}+v_{0} \frac{\partial n^{(2)}}{\partial x}+n_{0} \frac{\partial v^{(2)}}{\partial x}+\frac{\partial}{\partial x}\left(n^{(1)} v^{(1)}\right)=0, \\
& j^{2 \mathrm{D}(2)}=-e\left(n_{0} v^{(2)}+n^{(2)} v_{0}+n^{(1)} v^{(1)}\right) .
\end{aligned}
$$

In the Fourier representation, we come to the infinite system of algebraic equations:

$$
\begin{aligned}
& \left(\gamma-i\left[\omega_{p}-q_{m} v_{0}\right]\right) v_{m, p}^{(2)}+i \sum_{m^{\prime}, p^{\prime}} q_{m^{\prime}} v_{m^{\prime}, p^{\prime}}^{(1)} v_{m-m^{\prime}, p-p^{\prime}}^{(1)}= \\
& =-\frac{e}{m^{*}} E_{x, m, p}^{(2)}, \\
& \left(\omega_{p}-q_{m} v_{0}\right) n_{m, p}^{(2)}-q_{m} n_{0} v_{m, p}^{(2)}= \\
& =q_{m} \sum_{m^{\prime}, p^{\prime}} n_{m^{\prime}, p^{\prime}}^{(1)} v_{m-m^{\prime}, p-p^{\prime}}^{(1)}, \\
& j_{m, p}^{2 \mathrm{D}(2)}=-e\left(n_{0} v_{m, p}^{(2)}+n_{m, p}^{(2)} v_{0}+n_{m, p}^{(1)} v_{m, p}^{(1)}\right) .
\end{aligned}
$$

Eliminating the variable $n_{m, p}^{(2)}$ from the second equation of this system, we find the expression for a quadratic perturbation of the current. This quadratic perturbation is responsible for the emergence of the non-zero photocurrent:

$j_{m, p}^{2 \mathrm{D}(2)}=-\frac{e n_{0} \omega_{p}}{\omega_{p}-q_{m} v_{0}}\left(v_{m, p}^{(2)}+\sum_{m^{\prime}, p^{\prime}} n_{m^{\prime}, p^{\prime}}^{(1)} v_{m-m^{\prime}, p-p^{\prime}}^{(1)}\right)$.

Indeed, by definition, the photocurrent is determined as the result of the averaging over the spatial and temporal periods of an $a c$ current perturbation, i.e.,

$j^{\mathrm{ph}}=\left\langle j(x, t)-j_{0}\right\rangle_{x, t}=\left\langle j^{2 \mathrm{D}(1)}(x, t)+j^{2 \mathrm{D}(2)}(x, t)\right\rangle_{x, t}$.
In the case of monochromatic incident radiation, a linear perturbation of the current, $j^{2 \mathrm{D}(1)}(x, t) \sim$ $\sim \exp (-i \omega t)$, gives zero after the averaging. By performing the averaging,

$j^{\mathrm{ph}}=\left\langle j^{2 \mathrm{D}(2)}(x, t)\right\rangle_{x, t}=\int_{0}^{a} \frac{d x}{a} \int_{0}^{T} \frac{d t}{T} j^{2 \mathrm{D}(2)}(x, t)=j_{0,0}^{2 \mathrm{D}(2)}$,

we found that the photocurrent is equal to the zero Fourier harmonic of a quadratic perturbation of the current, $j_{0,0}^{2 \mathrm{D}(2)}$. The latter can be found from formula (19) in the limit $m \rightarrow 0$ and $p \rightarrow 0$. As a result, we have

$j_{0,0}^{2 \mathrm{D}(2)}=-e n_{0}\left(v_{0,0}^{(2)}+\sum_{m^{\prime}, p^{\prime}} n_{m^{\prime}, p^{\prime}}^{(1)} v_{-m^{\prime},-p^{\prime}}^{(1)}\right)$.

The first equation of system (18) yields

$v_{0,0}^{(2)}=-\frac{e}{m^{*} \gamma} E_{x, 0,0}^{(2)}-\frac{i}{\gamma} \sum_{m^{\prime}, p^{\prime}} q_{m^{\prime}} v_{m^{\prime}, p^{\prime}}^{(1)} v_{-m^{\prime},-p^{\prime}}^{(1)}$.

It is easy to verify that second term is identically zero. Indeed, passing to the summation over positive indices, we obtain

$$
\begin{aligned}
& \sum_{m^{\prime}, p^{\prime}} q_{m^{\prime}} v_{m^{\prime}, p^{\prime}}^{(1)} v_{-m^{\prime},-p^{\prime}}^{(1)}= \\
& =\sum_{\left(m^{\prime}, p^{\prime}\right)>0}\left(q_{m^{\prime}} v_{m^{\prime}, p^{\prime}}^{(1)} v_{-m^{\prime},-p^{\prime}}^{(1)}+q_{-m^{\prime}} v_{-m^{\prime},-p^{\prime}}^{(1)} v_{m^{\prime}, p^{\prime}}^{(1)}\right)=0 .
\end{aligned}
$$

Thus,

$v_{0,0}^{(2)}=-\frac{e}{m^{*} \gamma} E_{x, 0,0}^{(2)}$,

where $E_{x, 0,0}^{(2)}$ should be found from the electrodynamic equation (11) for the second order of perturbation theory. In the limit $m \rightarrow 0, p \rightarrow 0$, we have $\Delta_{0,0}=2$ and $\Lambda_{0,0}=1$ (see Appendix 1). As a result, Eq. (11) gives the following simple relation:

$E_{x, 0,0}^{(2)}=-\frac{2 \pi}{\sqrt{\epsilon_{I}} c} j_{0,0}^{2 \mathrm{D}(2)}$.

Here, we used that $j_{x, 0,0}^{G(2)}=0$. The latter reflects the apparent fact that the $d c$ current cannot flow across the grating strips.

Substituting Eq. (25) into Eq. (22) and taking Eq. (26) into account, we can obtain the expression for the photocurrent through quantities of the first order in the perturbation:

$j_{0,0}^{2 \mathrm{D}(2)}=-e \frac{\gamma}{\gamma+\Gamma} \sum_{m^{\prime}, p^{\prime}} n_{m^{\prime}, p^{\prime}}^{(1)} v_{-m^{\prime},-p^{\prime}}^{(1)}$,

ISSN 2071-0194. Ukr. J. Phys. 2017. Vol. 62, No. 10 
where the factor $\Gamma=2 \pi e^{2} n_{0} /\left(m^{*} c \sqrt{\epsilon_{I}}\right)$ has a meaning of the radiative decay rate. It should be noted that formula (27) contains the factor $\gamma /(\gamma+\Gamma)$, which was omitted in the previous theory [15]. The emergence of this factor is stipulated by the sequential consideration of Maxwell's equations in the second order of perturbation theory.

Using Eqs. (15) and (16), we obtain the final formula, which expresses the photocurrent through the spatial Fourier harmonics of the $a c$ electric field in the plane of 2DEG:

$j^{\mathrm{ph}}=-\frac{2 e^{3} n_{0}}{m^{2}} \frac{\gamma}{\gamma+\Gamma} \sum_{m} \frac{q_{m}}{\omega-q_{m} v_{0}} \frac{\left|E_{x, m, 1}^{(1)}\right|^{2}}{\gamma^{2}+\left(\omega-q_{m} v_{0}\right)^{2}}$.

On the derivation of the last equations in the case of a monochromatic wave, we used $E_{x, m, p}^{(1)} \sim \delta_{p, 1} \tilde{E}_{0}$ (see Appendix 2). This means that the sum over $p^{\prime}$ in Eq. (27) contains only terms corresponding to the frequency of the external signal, $\omega$. In the $d c$ current regime, the illumination of the plasmonic structure by terahertz radiation induces the additional $d c$ photovoltage:

$\delta U^{\mathrm{ph}}=-\mathcal{L} \frac{j^{\mathrm{ph}}}{\sigma_{0}}$,

where $\mathcal{L}$ is the channel length. This photovoltage is the measured quantity in experiments and is often mentioned as the photoresponse. The expression for the photoresponse,

$\delta U^{\mathrm{ph}}=\frac{2 e \gamma^{2} \mathcal{L}}{m^{*}(\gamma+\Gamma)} \sum_{m} \frac{q_{m}}{\omega-q_{m} v_{0}} \frac{\left|E_{x, m, 1}^{(1)}\right|^{2}}{\gamma^{2}+\left(\omega-q_{m} v_{0}\right)^{2}}$,

together with the solution of the electrodynamic problem Eq. (37) (see Appendix 2) finalize the formulation of the perturbation theory of the photoresponse in the hybrid plasmonic structures.

\section{Results and Discussions}

\subsection{Effect of the electron}

drift on the spectral characteristics of the plasmonic structure

For the better comprehension of the physics of the drifting mechanism of $\mathrm{THz}$-wave detection, we start our analysis from several phenomenological speculations.

ISSN 2071-0194. Ukr. J. Phys. 2017. Vol. 62, No. 10
So far as the photoresponse is proportional to the squared electric field amplitude in 2DEG, the maximal values of photoresponse are expected to be in the frequency range of the plasmon resonance. In such structures, the plasmon resonance is accompanied by a great enhancement of the electromagnetic field in the near-field zone of the grating, including the region of 2DEG [43], that manifests itself by the resonant absorption of an incident wave. Such absorption is identified as specific extrema in the transmission and reflection spectra [10,43-45].

In the absence of the electron drift $\left(E_{0}=0\right.$, $v_{0}=0$ ), the detected signal is equal to zero due to the symmetric contributions of the plasmons with positive and negative wavevectors [see formula (29)]. In this case, the minima corresponding to the wavevectors $q=2 \pi|m| / a$ can only be observed in transmission spectra.

Under the steady-state electron drift $\left(E_{0} \neq 0, v_{0} \neq\right.$ $\neq 0)$, the symmetry of the system is broken with respect to the mapping of $q_{m} \rightarrow q_{-m}$. This means that the Fourier harmonics of the electric fields, $E_{x, m, 1}^{(1)}$ and $E_{x,-m, 1}^{(1)}$, will be different, and their contributions to the photoresponse will not be compensated. Thus, the photocurrent increases with the asymmetry of plasmons with opposite signs of wavevectors.

The symmetry breaking also leads to the specific splitting of the plasmon resonance extrema in the transmission/reflection spectra. This splitting can be estimated with the use of the dispersion law of the drifting 2D plasmons that is obtained in the electrostatic approximation:

$\omega_{\mathrm{sc}}(q)=v_{0} q+\left.\omega_{\mathrm{sc}}(q)\right|_{v_{0}=0}$,

where $\left.\omega(q)_{\mathrm{sc}}\right|_{v_{0}=0}$ is the plasmon frequency in the absence of the electron drift. In this model, the splitting is determined by the Doppler shift, $v_{0} q$ (see, e.g., $[4,46,47])$. For the estimations, we used the expression of $\left.\omega_{\mathrm{sc}}(q)\right|_{v_{0}=0}$ in the case of $2 \mathrm{D}$ plasmons gated by the ideal metallic mirror:

$\left.\omega_{\mathrm{sc}}(q)\right|_{v_{0}=0}=\sqrt{\frac{4 \pi e^{2} n^{2 \mathrm{D}}|q|}{m^{*} \epsilon_{\mathrm{eff}}(q)}}$,

where $\epsilon_{\mathrm{eff}}(q)$ is the effective dielectric permittivity of the assumed plasmonic structure and is given by the formula

$\epsilon_{\mathrm{eff}}(q)=\epsilon_{I I} \operatorname{cth}|q| D+\epsilon_{I I I} \frac{\epsilon_{I}+\epsilon_{I I I} \operatorname{cth}|q| D_{s}}{\epsilon_{I I I}+\epsilon_{I} \operatorname{cth}|q| D_{s}}$. 


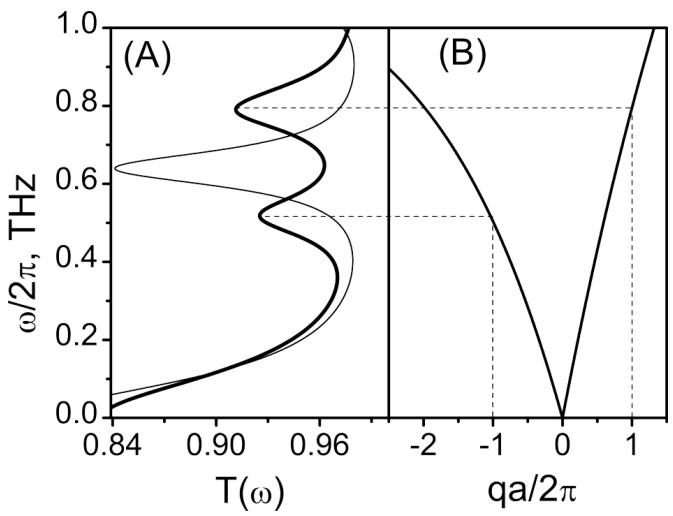

Fig. 2. Panel A: transmission spectrum of AlGaAs/GaAs hybrid plasmonic structure at $E_{0}=0 \mathrm{~V} / \mathrm{cm}$ (thin line) and $E_{0}=200 \mathrm{~V} / \mathrm{cm}$ (thick line). Panel B: dispersion curves of gated $2 \mathrm{D}$ plasmons calculated according to Eq. (30) for $v_{0}=7.5 \times 10^{6} \mathrm{~cm} / \mathrm{s}$. The parameter $\gamma=0.83 \times 10^{12} \mathrm{~s}^{-1}$ is obtained from the slope of $d c$ characteristics, $v_{0}\left(E_{0}\right)$ (see Fig. 5(B)). Geometric parameters of the structures are following: $a=0.5 \mu \mathrm{m}, b=0.4 \mu \mathrm{m}, D=40 \mathrm{~nm}$, and $D_{s}=1 \mu \mathrm{m}$. Dielectric constants: $\epsilon_{I}=1, \epsilon_{I I}=12.9, \epsilon_{I I I}=9.3\left(\mathrm{Al}_{2} \mathrm{O}_{3}\right.$ is assumed as a material for the substrate). Metallic grating is assumed to be a gold with a bulk conductivity of $4 \times 10^{17} \mathrm{~s}^{-1}$; thickness of strips $d_{g}=20 \mathrm{~nm}$, and $\sigma_{0}^{G}=8 \times 10^{11} \mathrm{~cm} \mathrm{~s}^{-1}$

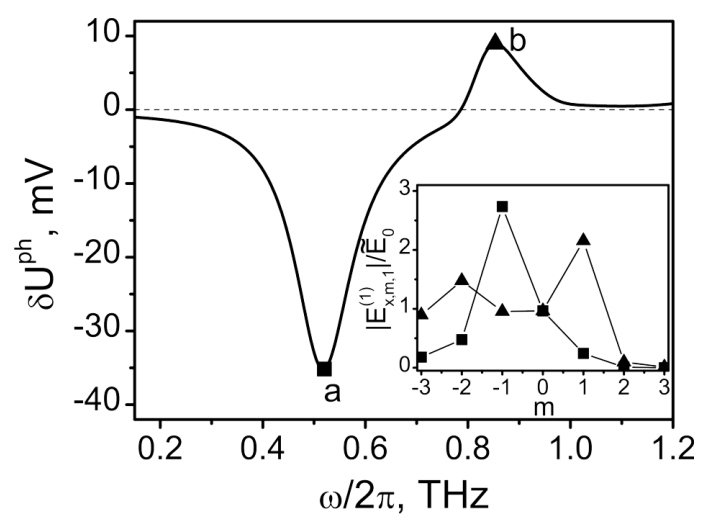

Fig. 3. Photoresponse spectrum of the assumed plasmonic structure. Points $a$ and $b$ mark the selected frequencies $\omega / 2 \pi=$ $=0.49,0.81 \mathrm{THz}$, for which the amplitudes of spatial Fourier harmonics $\left|E_{x, m, 1}^{(1)}\right|$ of the field in the plane of 2DEG for different $m$ are shown in the inset. Squares correspond to $\omega / 2 \pi=$ $=0.49 \mathrm{THz}$ and triangles correspond to $\omega / 2 \pi=0.81 \mathrm{THz}$. Power density of incident radiation is equal to $1 \mathrm{~W} / \mathrm{cm}^{2}$, the length of a $2 \mathrm{DEG}$ channel, $\mathcal{L}=2 \mathrm{~mm}$. Other parameters are the same as in Fig. 2

The more precise calculation of the drifting splitting of the plasmon resonance requires the cumbersome analysis of plasmon spectra under the grating including the effect of the branch folding $[10,12]$.
It should be noted that, for the distinct observation of the drifting mechanism of the plasmon resonance splitting, it is necessary to fulfil the number of requirements to the parameters of the plasmonic structure. One should use heterostructures with high electron mobilities, low electron concentrations, and as much as possible drift velocities. For example, such conditions can be realized in the plasmonic structures based on modern, high-quality AlGaAs/GaAs quantum heterostructures. Illustration of the drifting mechanism of plasmon resonance splitting in a transmission spectrum and its interrelation with the dispersion law (30) is demonstrated in Fig. 2 for $\mathrm{AlGaAs} / \mathrm{GaAs}$ heterostructure with the electron concentration $n_{0}=10^{11} \mathrm{~cm}^{-2}$ at the applied $d c$ field $E_{0}=200 \mathrm{~V} / \mathrm{cm}\left(v_{0}=7.5 \times 10^{6} \mathrm{~cm} / \mathrm{s}\right.$, taken from the calculation of $d c$ transport characteristics in Ref. [48]). As seen from Fig. 2 (A), the splitting of the plasmon resonance is $\sim 0.3 \mathrm{THz}$, and the positions of extrema are in good agreement with characteristic plasmon frequencies $\omega_{\mathrm{sc}}(-2 \pi / a)=2 \pi 0.5 \mathrm{THz}$ and $\omega_{\mathrm{sc}}(2 \pi / a)=2 \pi 0.8 \mathrm{THz}$. Such good matching is realized for metallic gratings with filling factors, $f=b / a$, closed to 1 . In the calculation, $f=0.8$.

\subsection{Photoresponse spectrum}

The spectrum of photoresponse of the plasmonic structure with the same parameters is shown in Fig. 3. As seen, the form of the spectrum has the wellpronounced extrema that correspond to the resonant frequencies in the transmission spectrum. The polarity of the detected signal depends, according to Eq. (29), on the sign of the wavenumber, $q_{m}$, of the excited plasmon mode. The extremum of the photoresponse that occurs at the frequency $\omega / 2 \pi=0.49 \mathrm{~Hz}$ has the value $\delta U^{\mathrm{ph}} \approx-40 \mathrm{mV}$ and corresponds to the excitation of the plasmon mode with $q_{-1}$. At the same time, the extremum at the frequency $\omega / 2 \pi=$ $=0.81 \mathrm{THz}$ has the positive sign, $\delta U^{\mathrm{ph}}=10 \mathrm{mV}$, and one associates with the excitation of the plasmon mode with $q_{+1}$. This is demonstrated in the inset in Fig. 3 by the dependences of the Fourier-harmonics $\left|E_{x, m, 1}^{(1)}\right|$ of the field in the plane of 2DEG vs number $m$. For the frequencies that correspond to the extrema of $\delta U^{\mathrm{ph}}$, the electric field in the plane of 2DEG has almost single-mode spatial structure, and the Fourier-harmonics $E_{x,-1,1}^{(1)}$ and $E_{x, 1,1}^{(1)}$ dominate. For the frequencies situated between the plasmon resonance frequencies, the spatial structure of the field 
can be much more complicated and is described by Fourier-harmonics of higher orders. In this case, the contributions of the different Fourier-harmonics can be compensated that leads to a decrease of the photoresponse.

\subsection{Analysis of the substrate effect}

The substrate is the constitutive part of a hybrid plasmonic structure. The presence of the optically thick substrate can essentially modify $\mathrm{THz}$ properties of the assumed structure, including the transmission/absorption and photoresponse spectra. The thick substrate can give rise to an essential suppression of the plasmon resonance. It relates to a decrease of the amplitude of the $a c$ electric field in the plane of 2DEG due to the effect of destructive interference of the two waves: transmitted wave and the wave reflected from the bottom interface of a substrate. For the efficient design of a $\mathrm{THz}$ detector, the thickness of a substrate should be chosen in such way that the frequencies of the Fabry-Perot and plasmon resonances will be close.

The spectra of $\delta U^{\mathrm{ph}}(\omega)$ are shown in Fig. $4(\mathrm{~A})$ for three types of substrates: membrane-like $\left(D_{s}=\right.$ $=1 \mu \mathrm{m})$, non-resonant $\left(D_{s}=46 \mu \mathrm{m}\right)$, and resonant $\left(D_{s}=92 \mu \mathrm{m}\right)$. As seen, the photoresponse in the case of non-resonant substrate is almost by three times less than in the cases of the membrane-like and resonant substrates. Such damping of the photoresponse is associated with a decrease of the amplitude of the $a c$ electric field in the plane of 2DEG and, as a result, the suppression of the plasmon resonance. This illustrates the spatial distribution of the quantity $\left|E_{x}(x, D)\right|$ (Fig. $\left.4(\mathrm{~B})\right)$ and absorption spectra $L(\omega)$ (inset to Fig. $4(\mathrm{~A})$ ). Consequently, the correct matching of the substrate parameters can completely compensate, in the defined frequency range, the negative effect of the destructive interference on the photoresponse magnitudes.

\subsection{Effect of electron heating}

In the majority of previous theories devoted to the electro-optical characterization of plasmon structures $[11,15]$, the momentum relaxation rate, $\gamma$, was assumed to be a constant quantity and independent of the magnitude of an applied $d c$ field. However, in the case of $\mathrm{A}^{I I I} \mathrm{~B}^{V}$ semiconductor compounds, the electron scattering can be strongly intensified with increasing $E_{0}$ due to the activation of the nonelas-
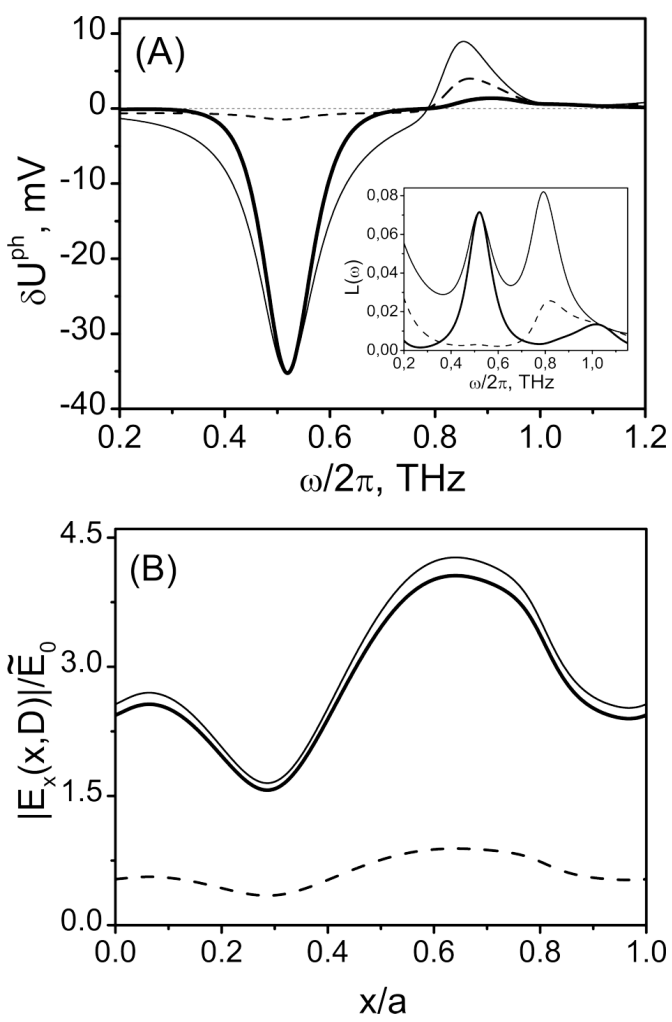

Fig. 4. Panel A: Photoresponse and absorption (inset) spectra of the plasmonic structure for various thicknesses of the substrate. Panel B: spatial distribution of the amplitude of the $x$-component of an electric field in the plane of 2DEG calculated in the linear approximation at $\omega / 2 \pi=0.57 \mathrm{THz}$. Thin, dashed, and thick lines correspond to $D_{s}=1,46$, and $92 \mu \mathrm{m}$. Other parameters are the same as in Fig. 2

tic electron-optical phonon interaction. This results in a sublinear behavior of current-voltage characteristics [49]. Thus, the rigorous modeling of $\mathrm{THz}$ detectors also requires the account for the effects of electron heating.

We used the field dependences of the drift velocity, $v_{0}\left(E_{0}\right)$, obtained from the kinetic theory of $2 \mathrm{D}$ electrons. The drift velocity and differential electron mobility were calculated with the use of solutions of the Boltzmann transport equation in the frame of the electron temperature approach [48]. In the calculations, we restrict ourselves by the consideration of a range of moderate applied $d c$ fields, where the dependence of $v_{0}\left(E_{0}\right)$ still shows the quasilinear behavior. The values of momentum scattering rates $\gamma$ were obtained from the differential mobility corresponding to the slope, $d v_{0} / d E_{0}$. 

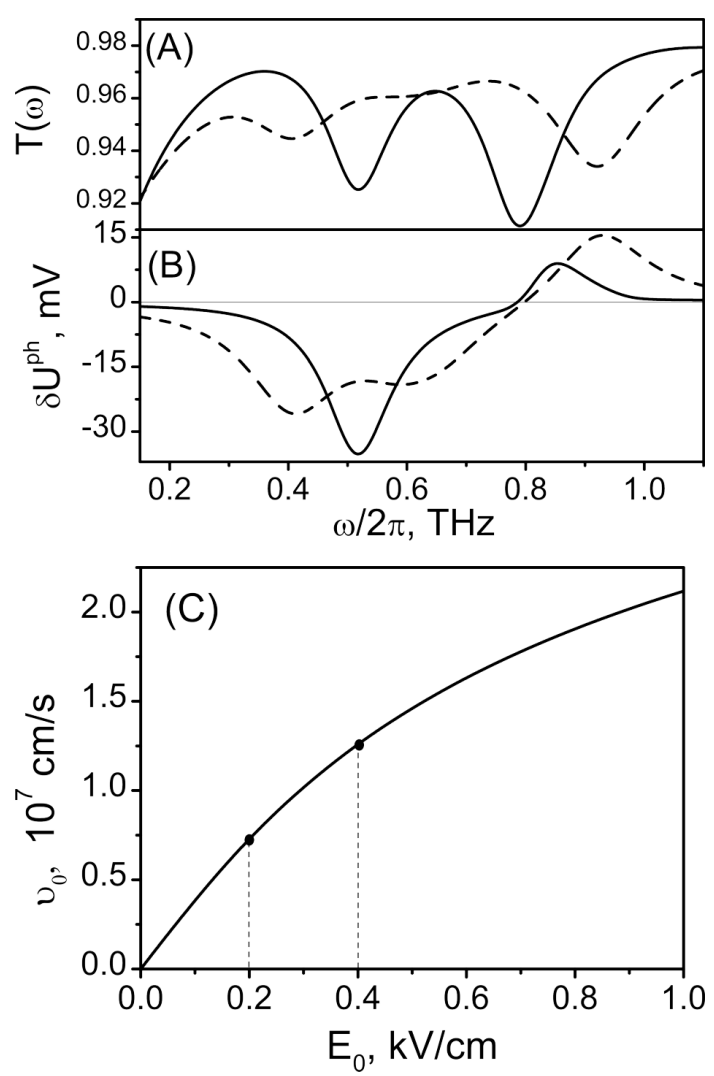

Fig. 5. Transmission spectra (panel A) and photoresponse spectra (panel B) of a plasmonic structure at $E_{0}=200 \mathrm{~V} / \mathrm{cm}$ (solid lines) and $E_{0}=400 \mathrm{~V} / \mathrm{cm}$ (dashed lines). Panel C: the drift velocity vs the $d c$ electric field for AlGaAs/GaAs quantum well at an ambient temperature of $77 \mathrm{~K}$. The selected values of $E_{0}$ are marked by points

The dependence of $v_{0}\left(E_{0}\right)$ is shown in Fig. $5(\mathrm{~B})$. With an increase of the applied $d c$ field from $200 \mathrm{~V} / \mathrm{cm}$ to $400 \mathrm{~V} / \mathrm{cm}$, the differential mobility decreases from $3.1 \times 10^{4} \mathrm{~cm}^{2} / \mathrm{Vs}\left(\gamma \sim 0.9 \mathrm{ps}^{-1}\right)$ to $2.2 \times 10^{4} \mathrm{~cm}^{2} / \mathrm{Vs}\left(\gamma \sim 1.3 \mathrm{ps}^{-1}\right)$. This is the cause for the increase of Ohmic losses in the plasmonic structure that is reflected in a decrease of the quality factor of the plasmon resonance and in a broadening of the photoresponse spectra (see Fig. $5(\mathrm{~A}, \mathrm{~B})$ ).

The increase of the magnitudes of $d c$ applied electric fields leads to several effects. The increase of the plasmon resonance splitting gives possibility of a fine tuning of the working frequency of a detector. However, simultaneously, the increase of the applied field gives rise to the increase of the electron scattering that decreases the quality factor of the plasmon resonance and, as a result, the photoresponse of a detec- tor. Thus, to keep the good frequency selectivity of the detector, the variation of the drift velocity should be restricted by a quasilinear interval.

\section{Summary}

In summary, the rigorous perturbation theory of the nonlinear response of a hybrid plasmonic structure with a drifting electron gas driven by the external electromagnetic field has been developed. In the frame of the consistent theory, the formula for the photoresponse is obtained and analyzed. The obtained formula contains the additional factor, which corresponds to the radiative decay rate. This important factor was missed in the previous theory [15]. The developed theory involves a multilayer geometry of the plasmonic structure and the effect of electron heating by the applied $d c$ electric field. The theory is applied to the analysis of the $\mathrm{THz}$ properties of the plasmonic structure based on quantum Al$\mathrm{GaAs} / \mathrm{GaAs}$ heterostructure with a low electron concentration of 2DEG of about $10^{11} \mathrm{~cm}^{-2}$.

It is shown that the form of the photoresponse spectrum is closely related to the specific splitting of the plasmon resonance induced by a strong electron drift. We have shown that, in the range of the applied $d c$ electric fields of $200 \ldots 400 \mathrm{~V} / \mathrm{cm}$, the photoresponse can reach the values of tens of $\mathrm{mV}$ for the external signals with a power density of $1 \mathrm{~W} / \mathrm{cm}^{2}$ in the frequency interval $0.3 \ldots 1 \mathrm{THz}$. We have revealed that the optically thick substrate can have the negative effect on the photoresponse due to the destructive interference of the waves transmitted and reflected from the bottom edge of a substrate. In this case, the coupling of the electromagnetic field with plasmon excitations can essentially decrease. The negative effect of the destructive interference can be avoided, by using a thin (membrane-like) substrate or the substrate, which matches the frequencies of the plasmon and Fabry-Perot resonances.

We have established that, at sufficiently strong applied electric fields, the quality factor of the plasmon resonances and magnitudes of the photoresponse can be essentially suppressed due to the effect of electron heating that is accompanied by an increase of the electron momentum scattering rate and Ohmic decay. Thus, the efficient design of $\mathrm{THz}$ detectors on the basis of hybrid plasmonic structures with drifting electron gas requires the fulfilment of several condi- 
tions. Particularly, it is necessary to use the metallic gratings with deeply submicron periods and quantum heterostructures with low electron concentrations. In addition, there are restrictions on the parameters of substrates and on a range of applied $d c$ electric fields.

In general, the developed theory can be thought as a step to the complete analysis of the nonlinear properties of hybrid plasmonic structures interacting with intense $\mathrm{THz}$ electromagnetic radiation.

The authors acknowledge the support of BMBF Project EUV-TERA with funding number 01DK17028. The authors are sincerely grateful to Dr.S.Danylyuk and Dr.S.Brose (TOS, RWTH Aachen University) for the discussion of various aspects of the paper and details of the planned experiments for the verification of the results of the proposed theory. The authors would like to express their gratitude to Prof. V.A. Kochelap (ISP NASU) for the valuable interest in this work.

\section{APPENDICES}

1. Boundary conditions and relations between the electric field and induced currents

The general solution of the wave equation (10) on $m, p$-Fourier harmonics of the x-component of the electric field takes the form:

$$
E_{x, m, p}^{(s)}(z)=\left\{\begin{array}{l}
A_{m, p}^{(s)} e^{k_{I, m, p} z}+\delta_{s, 1} \delta_{m, 0} \delta_{p, 1} \tilde{E}_{0} e^{-k_{I, m, p} z}, \\
z<0, \\
B_{m, p}^{(s)} e^{-k_{I I, m, p} z}+C_{m, p}^{(s)} e^{k_{I I, m, p} z}, \\
0<z<D, \\
H_{m, p}^{(s)} e^{-k_{I I I, m, p} z}+F_{m, p}^{(s)} e^{k_{I I I, m, p} z} \\
D<z<\mathfrak{D}, \\
t_{m, p}^{(s)} e^{-k_{I, m, p} z}, z>\mathfrak{D} .
\end{array}\right.
$$

Using the boundary conditions for the electric field and its derivative at three interfaces, $z \equiv h_{r}=\{0, D, \mathfrak{D}\} \quad(r=$ $=I, I I, I I I)$ :

$\left.E_{x, m, p}^{(s)}\right|_{z=h_{r}+0}=\left.E_{x, p, m}^{(s)}\right|_{z=h_{r}-0}$,

$\left.\frac{\epsilon_{r+1}}{k_{r+1, p, m}^{2}} \frac{\partial E_{x, p, m}^{(s)}}{\partial z}\right|_{z=h_{r}+0}-\left.\frac{\epsilon_{r}}{k_{r, p, m}^{2}} \frac{\partial E_{x, p, m}^{(s)}}{\partial z}\right|_{z=h_{r}-0}=$

$=\frac{4 \pi i}{\omega_{p}}\left[j_{p, m}^{G(s)} \delta_{r, I}+j_{p, m}^{2 \mathrm{D}(\mathrm{s})} \delta_{r, I I}\right]$,

where $\epsilon_{I V}=\epsilon_{I}$, we can obtain the system of the 6 algebraic equations for the determination of the coefficients $A_{m, p}^{(s)}, B_{m, p}^{(s)}$, $C_{m, p}^{(s)}, H_{m, p}^{(s)}, F_{m, p}^{(s)}$ and $t_{m, p}^{(s)}$. The solution of this system allows us to obtain expression (11), which connects $m, p$-Fourier harmonics of the $x$-component of the electric field in the plane of 2DEG with the corresponding Fourier harmonics of the currents and amplitude of the electric field of the incident electromagnetic wave. The above-introduced coefficients $\Lambda_{m, p}$ and
$\Delta_{m, p}$ are:

$\Delta_{m, p}=\left(1+\beta_{m, p}^{I I, I} \theta_{m, p}\right) \operatorname{ch} k_{I I, m, p} D+\left(\beta_{m, p}^{I I, I}+\theta_{m, p}\right) \operatorname{sh} k_{I I, m, p} D$,

$\Lambda_{m, p}=\operatorname{ch} k_{I I, m, p} \mathfrak{D}+\beta_{m, p}^{I, I I} \operatorname{sh} k_{I I, m, p} \mathfrak{D}$.

In the latter expressions

$\theta_{m, p}=\beta_{m, p}^{I I I, I I} \frac{\beta_{m, p}^{I, I I I}+\operatorname{th} k_{I I I, m, p} D_{s}}{1+\beta_{m, p}^{I, I I I} \operatorname{th} k_{I I I, m, p} D_{s}}$,

and

$\beta_{m, p}^{\left(r, r^{\prime}\right)}=\frac{\epsilon_{r} k_{r^{\prime}, m, p}}{\epsilon_{r^{\prime}} k_{r, m, p}}$.

In the limit $m \rightarrow 0, p \rightarrow 0, \beta_{0,0}^{\left(r, r^{\prime}\right)}=\sqrt{\epsilon_{r} / \epsilon_{r^{\prime}}}, \theta_{0,0}=\sqrt{\epsilon_{I} / \epsilon_{I I}}$, that gives $\Delta_{0,0}=2$ and $\Lambda_{0,0}=1$.

For any order of perturbation theory, it is useful to obtain the expression of $m, p$-Fourier harmonics of the $x$-component of the electric field in the grating plane:

$E_{x, m, p}^{(s)}(0)=2 \tilde{E}_{0} \delta_{m, 0} \delta_{p, 1} \frac{\delta_{s, 1} \Phi_{m, p}}{\Delta_{m, p}}-\frac{4 \pi i k_{I, m, p}}{\epsilon_{I} \omega_{p} \Delta_{m, p}} \times$

$\times\left(j_{m, p}^{G(s)} \Phi_{m, p}+j_{m, p}^{2 \mathrm{D}(\mathrm{s})}\right)$

with

$\Phi_{m, p}=\operatorname{ch} k_{I I, m, p} D+\theta_{m, p} \operatorname{sh} k_{I I, m, p} D$.

\section{Electrodynamics of the plasmon} structure in the linear approximation

Since, in the linear approximation $(s=1)$, the effect of the frequency multiplication does not arise, all temporal Fourier harmonics, except for $p=1$, are absent. From relations (11) and (34), using the Ohm law for the ac current in 2DEG, $j_{m, 1}^{2 \mathrm{D}(1)}=\sigma_{m, 1}^{2 \mathrm{D}} E_{x, m, 1}^{(1)}(D)$ (see Eq. (14)) and in the grating (4), which has the form $j_{m, 1}^{G(1)}=\sum_{m^{\prime}} \sigma_{m-m^{\prime}}^{G} E_{x, m^{\prime}, 1}^{(1)}(0)$ in the Fourier representation, we come to the infinite system of algebraic equations formulated for spatial $m$ - Fourier harmonics of the electric field in the grating plane:

$\sum_{m^{\prime}}\left[\delta_{m, m^{\prime}}+\frac{2 \pi i k_{I, m} W_{m}}{\epsilon_{I} \omega} \sigma_{m-m^{\prime}}^{G}\right] E_{x, m^{\prime}}(0)=W_{m} \delta_{m, 0} \tilde{E}_{0}$,

where

$W_{m}=2 /\left(1+\beta_{m}^{I I, I} Q_{m}\right)$,

$Q_{m}=\frac{\chi_{m}+\theta_{m}+\operatorname{th}\left(k_{I I, m} D\right)}{1+\left(\chi_{m}+\theta_{m}\right) \operatorname{th}\left(k_{I I, m} D\right)}$,

and

$\chi_{m}=\frac{4 \pi i k_{I I, m} \sigma_{m}^{2 \mathrm{D}}}{\epsilon_{I I} \omega}$.

For the compactness, the indices $s$ and $p$ were omitted. Fourier harmonics $E_{x, m}(0)$ allows us to find a spatial distribution of the $a c$ electric field in the structure and optical characteristics, including the transmission, reflection, and absorption 
spectra. Particularly, all coefficients of system (31) can be expressed through the quantity $E_{x, m}(0)$ as follows:

$$
\begin{aligned}
& A_{m}=E_{x, m}(0)-\delta_{m, 0} \tilde{E}_{0}, \\
& B_{m}=\frac{1}{2}\left(1+Q_{m}\right) E_{x, m}(0), \\
& C_{m}=\frac{1}{2}\left(1-Q_{m}\right) E_{x, m}(0), \\
& H_{m}=\frac{1}{2} e^{k_{I I I, m} D}\left[\left[1+\beta_{m}^{I I, I I I}\left(1-\chi_{m}\right)\right] B_{m} e^{-k_{I I, m} D}+\right. \\
& \left.+\left[1-\beta_{m}^{I I, I I I}\left(1+\chi_{m}\right)\right] C_{m} e^{k_{I I}, m}\right], \\
& F_{m}=\frac{1}{2} e^{-k_{I I I, m} D}\left[\left[1-\beta_{m}^{I I, I I I}\left(1-\chi_{m}\right)\right] B_{m} e^{-k_{I I, m} D}+\right. \\
& \left.+\left[1+\beta_{m}^{I I, I I I}\left(1+\chi_{m}\right)\right] C_{m} e^{k_{I I, m} D}\right], \\
& t_{m}=H_{m} e^{\left(k_{I, m}-k_{I I I, m}\right) \mathfrak{D}}+F_{m} e^{\left(k_{I, m}+k_{I I I, m}\right) \mathfrak{D} .}
\end{aligned}
$$

Having Eqs.(36), it is easy to find spatial Fourier harmonics of the electric field in the plane of 2DEG needed for calculations of the photoresponse:

$E_{x, m}(D) \equiv E_{x, m, 1}^{(1)}=E_{x, m}(0)\left[\operatorname{ch}\left(k_{I I, m} D\right)-Q_{m} \operatorname{sh}\left(k_{I I, m} D\right)\right]$,

and to calculate transmission, $T$, reflection, $R$, and absorption, $L$ coefficients:

$T=\left|t_{0} / \tilde{E}_{0}\right|^{2}, \quad R=\left|A_{0} / \tilde{E}_{0}\right|^{2}, \quad L=1-T-R$.

It should be noted that the solutions of Eqs. (35) have no convergence with respect to the rank of the system and give possibility to find only an approximate result [50]. Therefore, the usage of the direct Fourier method is ineffective from the point of view of the accuracy. This difficulty can be overcome with the use of the following procedure. System (35) can be rewritten in the coordinate representation in the form of a linear integral equation for the spatial distribution of the $x-$ component of the electric field:

$E_{x}(x, 0)=W_{0} \tilde{E}_{0}-\int_{-b / 2}^{b / 2} d x^{\prime} L\left(x, x^{\prime}\right) E_{x}\left(x^{\prime}, 0\right)$,

where $L\left(x, x^{\prime}\right)$ is the kernel of the integral operator:

$L\left(x, x^{\prime}\right)=\frac{2 \pi i}{\epsilon_{I} \omega} \frac{\sigma^{G}\left(x^{\prime}\right)}{a} \sum_{m} k_{I, m} W_{m} e^{i q_{m}\left(x-x^{\prime}\right)}$.

For the solutions of Eq. (39), Galerkin's scheme is used with the expansion of the electric field distribution in the strips in terms of orthogonal polynomials, $\left\{O_{l}\right\}, l=0 \ldots \infty$. The successful choice of the basis depends on the form of grating strips. For instance, for the assumed elliptic profile,

$\sigma^{G}(\tilde{x})=\sigma_{0}^{G} \begin{cases}\sqrt{1-\tilde{x}^{2}}, & -1 \leq \tilde{x} \leq 1, \\ 0, & 1 \leq \tilde{x} \leq 2 / f, \quad f=b / a,\end{cases}$

where $\tilde{x}=2 x / b$, the set of Chebyshev's polynomials was used as the basis. After the expansion of the field $E_{x}(\tilde{x}, 0)=$ $=\sum_{l=0}^{\infty} \mathcal{C}_{l} O_{l}(\tilde{x})($ at $|\tilde{x}|<1)$, Eq. (39) transforms to a system of algebraic equations for the coefficients $\mathcal{C}_{l}$

$\sum_{l^{\prime}=0}^{\infty} M_{l, l^{\prime}} \mathcal{C}_{l^{\prime}}=W_{0} \tilde{E}_{0} \delta_{l, 0}$,

$M_{l, l^{\prime}}=\delta_{l, l^{\prime}}+\frac{2 i f \sigma_{0}^{G}}{\omega \epsilon_{I}} \sum_{m} k_{I, m} W_{m} \zeta_{l, m} \zeta_{l^{\prime}, m}^{*}$,

$\zeta_{l, m}=\int_{-1}^{1} \sqrt{1-\tilde{x}^{2}} O_{l}(\tilde{x}) e^{-i \pi m f \tilde{x}} d \tilde{x}$.

The projection of the integral equation (39) on Chebyshev polynomials of the second kind results in the convergent matrix equation (42). The cause lies in the fact that such successful basis functions perform the analytic inversion of the singular part of the operator in (39) [51]. Finding the coefficients $\mathcal{C}_{l}$, we can express the Fourier harmonics of the field in the grating plane as follows:

$E_{x, m}(0)=W_{0} \tilde{E}_{0} \delta_{m, 0}-\frac{i \pi f \sigma_{0}^{G}}{\omega \epsilon_{I}} k_{I, m} W_{m} \sum_{l} \mathcal{C}_{l} \zeta_{l, m}$.

This allows us to calculate, as was mentioned above, all electrodynamic characteristics of the plasmonic structure in the first order of perturbation theory. Eventually, we note that the application of the Galerkin scheme to the analysis of effectively 2 D plasmonic structures can be found in Refs. [10,52].

1. M. Tonouchi. Cutting-edge terahertz technology. Nature Photonics 1, 97 (2007).

2. F. Sizov, A. Rogalski. THz detectors. Prog. Quant. Electron. 34, 278 (2010).

3. T. Otsuji, H. Karasawa, T. Watanabe, T. Suemitsu, M. Suemitsu, E. Sano, W. Knap, V. Ryzhii. Emission of terahertz radiation from two-dimensional electron systems in semiconductor nano-heterostructures. C. R. Physique 11, 421 (2010).

4. A.V. Chaplik. Absorption and emission of electromagnetic waves by two-dimensional plasmons. Surf. Sci. Rep. 5, 289 (1985).

5. V. Jakštas, I. Grigelionis, V. Janonis, G. Valušis, I. Kašalynas, G. Seniutinas, S. Juodkazis, P. Prystawko, M. Leszczyński. Electrically driven terahertz radiation of 2DEG plasmons in AlGaN/GaN structures at $110 \mathrm{~K}$ temperature. Appl. Phys. Lett. 110, 202101 (2017).

6. M.V. Krasheninnikov, A.V. Chaplik. Radiative decay of two-dimensional plasmons. Sov. Phys. JETP 61, 75 (1985).

7. M. Dyakonov, M. Shur. Shallow water analogy for a ballistic field effect transistor: New mechanism of plasma wave generation by dc current. Phys. Rev. Lett. 71, 2465 (1993).

8. M.Ali Khorrami, S. El-Ghazaly, S.-Q. Yu, H. Naseem. Terahertz plasmon amplification using two-dimensional electron-gas layers. J. Appl. Phys. 111, 094501 (2012).

9. O. Sydoruk. Drifting plasmons in open two-dimensional channels: modal analysis. J. Phys. D: Appl. Phys. 46, 135103 (2013).

ISSN 2071-0194. Ukr. J. Phys. 2017. Vol. 62, No. 10 
10. S.A. Mikhailov. Tunable solid-state far-infrared sources: New ideas and prospects. Recent Res. Devel. Appl. Phys. 2, 65 (1999).

11. S.A. Mikhailov. Plasma instability and amplification of electromagnetic waves in low-dimensional electron systems. Phys. Rev. B 58, 1517 (1998).

12. A.S. Petrov, D. Svintsov, V. Ryzhii, M.S. Shur. Amplifiedreflection plasmon instabilities in grating-gate plasmonic crystals. Phys. Rev. B 95, 045405 (2017).

13. M. Dyakonov, M. Shur. Plasma wave electronics: Novel terahertz devices using two dimensional electron fluid. IEEE Trans. Electron. Dev. 43, 1640 (1996).

14. G.R. Aizin, D.V. Fateev, G.M. Tsymbalov, V.V. Popov. Terahertz plasmon photoresponse in a density modulated two-dimensional electron channel of a GaAs/AlGaAs fieldeffect transistor. Appl. Phys. Lett. 91, 163507 (2007).

15. G.R. Aizin, V.V. Popov, O.V. Polischuk. Plasmon enhanced electron drag and terahertz photoconductance in a grating-gated field-effect transistor with two-dimensional electron channel. Appl. Phys. Lett. 89, 143512 (2006).

16. W. Knap, Y. Deng, S. Rumyantsev, J.-Q. Lü, M.S. Shur, C.A. Saylor, L.C. Brunel. Resonant detection of subterahertz radiation by plasma waves in a submicron field-effect transistor. Appl. Phys. Lett. 80, 3433 (2002); W. Knap, Y. Deng, S. Rumyantsev, M.S. Shur. Resonant detection of subterahertz and terahertz radiation by plasma waves in submicron field-effect transistors. Appl. Phys. Lett. 81, 4637 (2002).

17. H. Marinchio, C. Palermo, A. Mahi, L. Varani, V. Korotyeyev. External excitation of hybrid plasma resonances in a gated semiconductor slab: An analytical study. J. Appl. Phys. 116, 013707 (2014).

18. K.Y. Xu, X.F. Lu, A.M. Song, G. Wang. Enhanced terahertz detection by localized surface plasma oscillations in a nanoscale unipolar diode. J. Appl. Phys. 103, 113708 (2008).

19. J. Torres, P. Nouvel, A. Penot, L. Varani, P. Sangar, B. Grimbert, M. Faucher, G. Ducournau, C. Gaqui, I. Iniguez-de-la-Torre, J. Mateos, T. Gonzalez. Nonlinear nanochannels for room temperature terahertz heterodyne detection. Semicond. Sci. Technol. 28, 125024 (2013).

20. W. Knap, V. Kachorovskii, Y. Deng, S. Rumyantsev, J.-Q. Lu, R. Gaska, M.S. Shur, G. Simin, X. Hu, M. Asif Khan, A. Saylor, L.C. Brunel. Nonresonant detection of terahertz radiation in field effect transistors. J. Appl. Phys. 91, 9346 (2002).

21. W. Knap, F. Teppe, Y. Meziani, N. Dyakonova, J. Lusakowski, F. Boeuf, T. Skotnicki, D. Maude, S. Rumyantsev, M.S. Shur. Plasma wave detection of sub-terahertz and terahertz radiation by silicon field-effect transistors. Appl. Phys. Lett. 85, 675 (2004).

22. M. Sakowicz, M. B. Lifshits, O. A. Klimenko, F. Schuster, D. Coquillat, F. Teppe, W. Knap. Terahertz responsivity of field effect transistors versus their static channel conductivity and loading effects. J. Appl. Phys. 110, 054512 (2011).

ISSN 2071-0194. Ukr. J. Phys. 2017. Vol. 62, No. 10
23. S. Preu, S. Kim, R. Verma, P.G. Burke, M.S. Sherwin, A.C. Gossard. An improved model for non-resonant terahertz detection in field-effect transistors. J. Appl. Phys. 111, 024502 (2012).

24. R. Tauk, F. Teppe, S. Boubanga, D. Coquillat, W. Knap, Y.M. Meziani, C. Gallon, F. Boeuf, T. Skotnicki, C. Fenouillet-Beranger, D.K. Maude, S. Rumyantseva, M.S. Shur. Plasma wave detection of terahertz radiation by silicon field effects transistors: Responsivity and noise equivalent power. Appl. Phys. Lett. 89, 253511 (2006).

25. A. Lisauskas, U. Pfeiffer, E. Öjefors, P.H. Bolivar, D. Glaab, H.G. Roskos. Rational design of high-responsivity detectors of terahertz radiation based on distributed self-mixing in silicon field-effect transistors. J. Appl. Phys. 105, 114511 (2009).

26. F. Schuster, D. Coquillat, H. Videlier, M. Sakowicz, F. Teppe, L. Dussopt, B. Giffard, T. Skotnicki, W. Knap. Broadband terahertz imaging with highly sensitive silicon CMOS detectors. Opt. Express 18, 7827 (2011).

27. J.-Q. Lü, M.S. Shur, J.L. Hesler, L. Sun, R. Weike. Terahertz detector utilizing two-dimensional electronic fluid. IEEE Electron. Dev. Lett. 19, 373 (1998).

28. A. El Fatimy, F. Teppe, N. Dyakonova, D. Seliuta, G. Valušis, A. Shchepetov, Y. Roelens, S. Bollaert, A. Cappy, S. Rumyantsev. Resonant and voltage-tunable terahertz detection in InGaAs/InP nanometer transistors. Appl. Phys. Lett. 89, 131926 (2006).

29. S. Boubanga-Tombet, F. Teppe, D. Coquillat, S. Nadar, N. Dyakonova, H. Videlier, W. Knap, A. Shchepetov, C. Gardes, Y. Roelens, S. Bollaert, D. Seliuta, R. Vadoklis, G. Valušis. Current driven resonant plasma wave detection of terahertz radiation: Toward the Dyakonov-Shur instability. Appl. Phys. Lett. 92, 212101 (2008).

30. T.A. Elkhatib, V.Yu. Kachorovskii, W.J. Stillman, S. Rumyantsev, X.-C. Zhang, M.S. Shur. Terahertz response of field-effect transistors in saturation regime. Appl. Phys. Lett. 98, 243505 (2011).

31. S. Danylyuk, P. Loosen, K. Bergmann, H. Kim, L. Juschkin. Scalability limits of Talbot lithography with plasmabased extreme ultraviolet sources. J. Micro/Nanolith. MEMS MOEMS 12 (3), 033002 (2013).

32. G. Kunkemöller, T.W.W. Maß, A.-K.U. Michel, Hyun-Su Kim, S. Brose, S. Danylyuk, Th. Taubner, L. Juschkin. Extreme ultraviolet proximity lithography for fast, flexible and parallel fabrication of infrared antennas. Opt. Express 23 (20), 25487 (2015).

33. X.G. Peralta, S.J. Allen, M.C. Wanke, N.E. Harff, J.A. Simmons, P. Lilly, J.L. Reno, P.J. Burke, J.P. Eisenstein. Terahertz photoconductivity and plasmon modes in double-quantum-well field-effect transistors. Appl. Phys. Lett. 81, 1627 (2002).

34. E.A. Shaner, M. Lee, M.C. Wanke, A.D. Grine, J.L. Reno, S.J. Allen. Single-quantum-well grating-gated terahertz plasmon detectors. Appl. Phys. Lett. 87, 193507 (2005). 
35. D.M. Yermolayev, K.M. Marem'yanin, D.V. Fateev, S.V. Morozov, N.A. Maleev, V.E. Zemlyakov, V.I. Gavrilenko, S.Yu. Shapoval, F.F. Sizov, V.V. Popov. Terahertz detection in a slit-grating-gate field-effect-transistor structure. Solid-State Electronics 86, 64 (2013).

36. N. Nader Esfahani, R.E. Peale, W.R. Buchwald, C.J. Fredricksen, J.R. Hendrickson, J.W. Cleary. Millimeter-wave photoresponse due to excitation of two-dimensional plasmons in InGaAs/InP high-electron-mobility transistors. $J$. Appl. Phys. 114, 033105 (2013).

37. V.V. Popov, D.V. Fateev, T. Otsuji, Y.M. Meziani, D. Coquillat, W. Knap. Plasmonic terahertz detection by a double-grating-gate field-effect transistor structure with an asymmetric unit cell. Appl. Phys. Lett. 99, 243504 (2011).

38. V.V. Popov, D.V. Fateev, O.V. Polischuk, M.S. Shur. Enhanced electromagnetic coupling between terahertz radiation and plasmons in a grating-gate transistor structure on membrane substrate. Opt. Express 18, 16771 (2010).

39. Yu.M. Lyaschuk, V.V. Korotyeyev. Interaction of terahertz electromagnetic field with metallic grating: Nearfield zone. Ukr. J. Phys. Opt. 13, 142 (2012).

40. M.A. Ordal, L.L. Long, R.J. Bell, S.E. Bell, R.R. Bell, R.W. Alexander C.A. Ward. Optical properties of the metals $\mathrm{Al}, \mathrm{Co}, \mathrm{Cu}, \mathrm{Au}, \mathrm{Fe}, \mathrm{Pb}, \mathrm{Ni}, \mathrm{Pd}, \mathrm{Pt}, \mathrm{Ag}, \mathrm{Ti}$, and $\mathrm{W}$ in the infrared and far infrared. Appl. Optics 22 (7), 1099 (1983).

41. O.V. Shapoval, R. Sauleau, A.I. Nosich. Scattering and absorption of waves by flat material strips analyzed using generalized boundary conditions and nystrom-type algorithm. IEEE Trans. Antennas Propag. 59, 3339 (2011).

42. I.O. Sukharevsky, O.V. Shapoval, A. Altintas, A.I. Nosich. Validity and limitations of the median-line integral equation technique in the scattering by material strips of subwavelength thickness. IEEE Trans. Antennas Propag. 62, 3623 (2014).

43. Yu.M. Lyaschuk, V.V. Korotyeyev. Interaction of a terahertz electromagnetic wave with the plasmonic system "grating-2D-gas". Analysis of features of the near field. Ukr. J. Phys. 59, 495 (2014).

44. S.J. Allen, Jr., D.C. Tsui, R.A. Logan. Observation of the two-dimensional plasmon in silicon inversion layers. Phys. Rev. Lett. 38 (17), 980 (1977).

45. Hua Qin, Yao Yu, Xiang Li, Jiandong Sun, Yongdan Huang. Excitation of terahertz plasmon in two-dimensional electron gas. Terahertz Science and Technology 9 (2), 71 (2016).

46. R.E. Tyson, R.J. Stuart, H.P. Hughes, J.E.F. Frost, D.A. Ritchie, G.A.C. Jones, C. Shearwood. Non-linear Doppler shift of the plasmon resonance in a grating-coupled drifting 2DEG. Int. J. Infrared Millimeter Waves 14, 1237 (1993).

47. A.S. Bhatti, D. Richards, H.P. Hughes, D.A. Ritchie. Spatially resolved Raman scattering from hot acoustic and optic plasmons. Phys. Rev. B 53, 11016 (1996).
48. V.V. Korotyeyev. Theory of high-field electron transport in the heterostructures $\mathrm{Al}_{x} \mathrm{Ga}_{1-x} \mathrm{As} / \mathrm{GaAs} / \mathrm{Al}_{x} \mathrm{Ga}_{1-x}$ with delta-doped barriers. Effect of real-space transfer. Semiconductor Physics, Quantum Electronics \& Optoelectronics 18, 1 (2015).

49. W.T. Masselink. Electron velocity in GaAs: Bulk and selectively doped heterostructures. Semicond. Sci. Technol. 4, 503 (1989).

50. T.L. Zinenko, A.I. Nosich, Y. Okuno. Plane wave scattering and absorption by resistive-strip and dielectric-strip periodic gratings. IEEE Trans. Antennas Propag. 46 (10) 1498 (1998).

51. A.I. Nosich. Method of analytical regularization in computational photonics. Radio Sci. 51, 1421 (2016).

52. O.R. Matov, O.V. Polischuk, V.V. Popov. Electromagnetic emission from two-dimensional plasmons in a semiconductor-dielectric structure with metal grating: Rigorous theory. Int. J. Infrared Millimeter Waves 14 (7), 1455 (1993).

Received 18.09.17

\section{Ю.М. Лящук, В.В. Коротєєе}

\section{ТЕОРІЯ ДЕТЕКТУВАННЯ ТЕРАГЕРЦОВОГО ЕЛЕКТРОМАГНІТНОГО ВИПРОМІНЮВАННЯ В ГІБРИДНИХ ПЛАЗМОННИХ СТРУКТУРАХ 3 ДРЕЙФУЮЧИМ ЕЛЕКТРОННИМ ГАЗОМ}

Р е з ю м е

Побудована пертурбаційна теорія нелінійної взаємодії електромагнітного випромінювання з гібридною плазмонною структурою, яка являє собою двовимірну квантову гетероструктуру, інтегровану з плазмонним елементом у вигляді металічної гратки. Зокрема, проаналізовано нелінійний ефект детектування надвисокочастотного випромінювання дрейфуючим двовимірним електронним газом. На основі самоузгодженого розв'язку системи рівнянь Максвелла і нелінійних рівнянь гідродинаміки в другому порядку теорії збурень отриманий вираз для напруги фотовідгуку. Показано, що послідовне врахування поправок другого порядку теорії збурень в рівняннях Максвелла приводить до появи у виразі фотовідгуку додаткового фактора, який враховує радіаційні втрати. Теорія була застосована до аналізу ТГц властивостей плазмонної структури на основі квантової AlGaAs/GaAs гетероструктури. Проаналізовано вплив оптично товстої діелектричної підкладки та ефекту розігріву носіїв при високих дрейфових швидкостях на спектральні характеристики коефіцієнтів пропускання/поглинання та форму спектрів напруги фотовідгуку. Розроблено рекомендації щодо раціонального проектування детекторів ТГц випромінювання на основі гібридних плазмонних структур. 